\title{
Radio emission from the high-mass X-ray binary BP Crucis First detection
}

\author{
M. Pestalozzi ${ }^{1}$, U. Torkelsson ${ }^{1}$, G. Hobbs ${ }^{2}$, and Á. R. López-Sánchez ${ }^{2}$ \\ 1 Department of Physics, University of Gothenburg, 41296 Göteborg, Sweden \\ e-mail: [torkel;michele.pestalozzi]@physics.gu.se \\ 2 Australia Telescope National Facility, PO Box 76, Epping, NSW 1710, Australia \\ e-mail: [George.Hobbs; Angel.Lopez-Sanchez]@csiro.au
}

Received 20 August 2009 / Accepted 12 September 2009

\begin{abstract}
Context. BP Cru is a well known high-mass X-ray binary consisting of a late B hypergiant (Wray 977) and a neutron star, also observed as the X-ray pulsar GX301-2. No information about emission from BP Cru in bands other than X-rays and optical has been reported. A massive X-ray binaries containing black holes can produce radio emission from a jet.

Aims. To assess the presence of a radio jet, we searched for radio emission from BP Cru using the Australia Compact Array Telescope as part of a survey for radio emission from Be/X-ray transients.

Methods. We probed the $41.5 \mathrm{~d}$ orbit of BP Cru with the Australia Telescope Compact Array, close to both periastron and apastron. Results. BP Cru was clearly detected in our data on 4, possibly 6, of 12 occasions at 4.8 and $8.6 \mathrm{GHz}$. Our data suggest that the spectral index of the radio emission is modulated either by the X-ray flux or the orbital phase of the system.

Conclusions. We propose that the radio emission of BP Cru probably originates in two components: a persistent component, related to the mass donor Wray 977, and a periodic component, connected to accretion onto the neutron star, possibly originating from a (weak and short lived) jet.
\end{abstract}

Key words. X-ray: binaries - radio continuum: stars - stars: individual: BP Cru - stars: binaries: close - stars: supergiants

\section{Introduction}

The majority of high-mass X-ray binaries (HMXB) are classified as $\mathrm{Be} / \mathrm{X}$-ray transients, i.e., the compact object in these systems is the companion of a Be-star. Some Be/X-ray transients display X-ray outbursts at most of the periastron passages as the result of mass transfer from the Be-star. Another important group of $\mathrm{HMXBs}$ consists of systems with the mass donor that is a OB super- or hypergiant, that loses mass by means of a massive stellar wind, from which the compact object accretes mass.

The wind accreting X-ray pulsar GX301-2 (also known as $4 \mathrm{U}$ 1223-62) is part of the well known high-mass X-ray binary BP Cru. This system is peculiar in several aspects (Table 1): it contains both the most massive star known in these systems and among the slowest of pulsars.

The orbital period of BP Cru is $P_{\text {orb }}=41.5 \mathrm{~d}$, and the X-ray flares occur about two days before periastron (Watson et al. 1982). In addition, BP Cru exhibits a weaker X-ray flare close to apastron. The strong X-ray outbursts of BP Cru occur close to periastron making it similar to the Be/X-ray transients. Models of fast, dense stellar winds from the primary have difficulty in reproducing the correlations of the weak and strong X-ray outbursts with the orbital phases of BP Cru. Among the alternative models are those that incorporate a circumstellar disc around Wray 977 through which the neutron star passes at every periastron, an enhancement of the stellar wind close to periastron (Pravdo et al. 1995), as well as a spiral-type tidal stream of gas trailing the mass donor's rotation (Leahy \& Kostka 2008) that can produce a transient accretion disc around the neutron star, which explains the episodes of rapid spin-up of the X-ray pulsar (Koh et al. 1997).

Although Be/X-ray transients are easily observed in the $\mathrm{X}$-rays and some of them have known optical counterparts, very few radio observations of these objects have been reported in the literature. The radio surveys of X-ray binaries by Duldig et al. (1979) and Nelson \& Spencer (1988) report only upper limits for $7 \mathrm{Be} / \mathrm{X}$-ray transients, and one positive detection of $14 \mathrm{mJy}$ at $2 \mathrm{~cm}$ (April 1978) for A 1118-61 (Duldig et al. 1979). For this source, they found only upper limits at the $3 \mathrm{mJy}$ level in 7 other epochs. The orbital phases of the system during these observations are unclear. The main problem for the observation of these systems is the transient nature of the outbursts and, in some cases, the coarse X-ray positions.

Some other X-ray binaries (as e.g., microquasars) have relativistic jets that produce synchrotron emission at radio wavelengths. In general, microquasars are black hole candidates, but in a few cases the compact object might rather be a neutron star. The neutron star systems that are emitting radio waves tend to have high luminosities and weakly magnetised neutron stars. On the other hand, X-ray pulsars that contain strongly magnetised neutron stars but are less luminous, are as a rule not detected at radio wavelengths.

Outbursts in microquasars are characterised by an X-ray outburst close to periastron followed by the formation of a radio jet at a later time, when the X-ray spectrum indicates that the inner part of the accretion disc has vanished (Harmon et al. 1995). The 
Table 1. Characteristics of the BP Cru system.

\begin{tabular}{ll}
\hline \hline BP Cru & \\
\hline RA(J2000) 12:26:37.6 & Dec(J2000)-62:46:14 \\
Orbital period & $41.5 \mathrm{~d}$ \\
\hline Wray 977 & \\
\hline Mass & $39-68 M_{\odot}$ \\
Radius & $62 R_{\odot}$ \\
$T_{\text {eff }}$ & $18100 \mathrm{~K}$ \\
Luminosity & $5 \times 10^{5} L_{\odot}$ \\
Mass-loss rate & $10^{-5} M_{\odot} \mathrm{yr}^{-1}$ \\
Wind velocity & $305 \mathrm{~km} \mathrm{~s}^{-1}$ \\
\hline GX301-2 $^{\text {Mass }}{ }^{a}$ & \\
Spin period & $1.85 M_{\odot}$ \\
Surface magnetic field $^{b}$ & $696 \mathrm{~s}$ \\
\hline
\end{tabular}

${ }^{a}$ Kaper et al. 2006; ${ }^{b}$ Kreykenbohm et al. 2004; ${ }^{c}$ White et al. 1976.

radio outburst can therefore be delayed by up to some 10 days relative to the X-ray outburst.

With this in mind, we devised an observational programme to search for radio emission from $\mathrm{Be} / \mathrm{X}$-ray transients and other HMXBs that have semi-regular X-ray outbursts close to periastron. Our strategy was to distribute "snap-shot" observations to cover the orbital phases between periastron and apastron.

In this Letter, we present the first results of our observational campaign, the detection of radio continuum emission in the centimetre band at two frequencies $(4.8$ and $8.6 \mathrm{GHz})$ from the BP Cru system.

\section{Observations and data reduction}

Observations of BP Cru were completed at 12 epochs for a total of approximately $20 \mathrm{~h}$ of observations, 13 of which were on target. The observation epochs were spread between November 2008 and February 2009, covering almost four complete orbits. Every epoch consisted of about $1.5 \mathrm{~h}$ of data recording, and this time was divided between flux and phase calibrators and the target. The original strategy was to spread our observations over different orbital phases, from shortly before periastron to close to apastron. Our proposed schedule was then folded with the telescope availability to give the final schedule summarised in Table 2 and graphically presented in Fig. 1.

We used the Australia Telescope Compact Array (ATCA) in the 4.8 and $8.6 \mathrm{GHz}$ continuum bands in two linear polarisations. Data were recorded at the widest bandwidth, $128 \mathrm{MHz}$, spread over 33 channels per polarisation and frequency. All four polarisation products were recorded (XX, YY, XY, YX) in order to retrieve information about the level of polarisation of a detection. The time in every observation block was divided between a phase calibrator (1148-671), and the target, alternating $2 \mathrm{~min}$ scans on the calibrator with $6-8 \mathrm{~min}$ on the target. At the beginning of each run, a bandpass calibrator was also observed for some 5-10 min. Since the project was a detection experiment, no requirements were made as to which configuration ATCA had to be in at the different epochs. This had the advantage of greatly improving the scheduling flexibility but the disadvantage of having to reduce data in all kinds of configurations. In this respect, data at epochs 4-6, 11, and 12 (see Table 2) were found to be less useful for our program because they were recorded in the most compact ATCA configurations (see Table 2) and are hence affected significantly by Galactic emission, since BP Cru lies in the plane of the Galaxy. The $1 \sigma \mathrm{rms}$ level in those maps was on
Table 2. Details of the observations presented in this paper.

\begin{tabular}{ccccc}
\hline \hline Epoch & Date $(\mathrm{UT})^{a}$ & T-on [min] $^{b}$ & Phase $^{c}$ & Array $^{d}$ \\
\hline 1 & $2008-11-01,2212$ & 36 & -7 & $6 \mathrm{~A}$ \\
2 & $2008-11-05,1708$ & 54 & -3 & $6 \mathrm{~A}$ \\
3 & $2008-11-09,0443$ & 63 & +1 & $6 \mathrm{~A}$ \\
4 & $2008-11-18,0314$ & 45 & +10 & EW367 \\
5 & $2008-11-24,2055$ & 45 & +16 & EW367 \\
6 & $2008-12-16,1936$ & 54 & -4 & $750 \mathrm{~B}$ \\
7 & $2008-12-21,1840$ & 54 & +1 & $6 \mathrm{C}$ \\
8 & $2009-01-05,1719$ & 153 & +16 & $6 \mathrm{C}$ \\
9 & $2009-01-26,1603$ & 63 & -4 & $1.5 \mathrm{C}$ \\
10 & $2009-01-30,1415$ & 72 & +0 & $1.5 \mathrm{C}$ \\
11 & $2009-02-08,1936$ & 72 & +9 & EW352 \\
12 & $2009-02-15,1122$ & 54 & +16 & EW352 \\
\hline
\end{tabular}

${ }^{a}$ Times indicate the beginning of every observation.

${ }^{b}$ Effective time spent on-source.

${ }^{c}$ Days before (-) or after $(+)$ periastron. Periastron passage occurred at 2008-11-08, 2008-12-20, and 2009-01-30 (see Koh et al. 1997).

$d$ ATCA configuration for the observations. When including all antennae, the $u v$-coverage from the "6"-configurations is regular, from the "1.5"-configurations slightly bimodal and from the EW- and 750 -configurations (compact configurations) it is strongly bimodal.

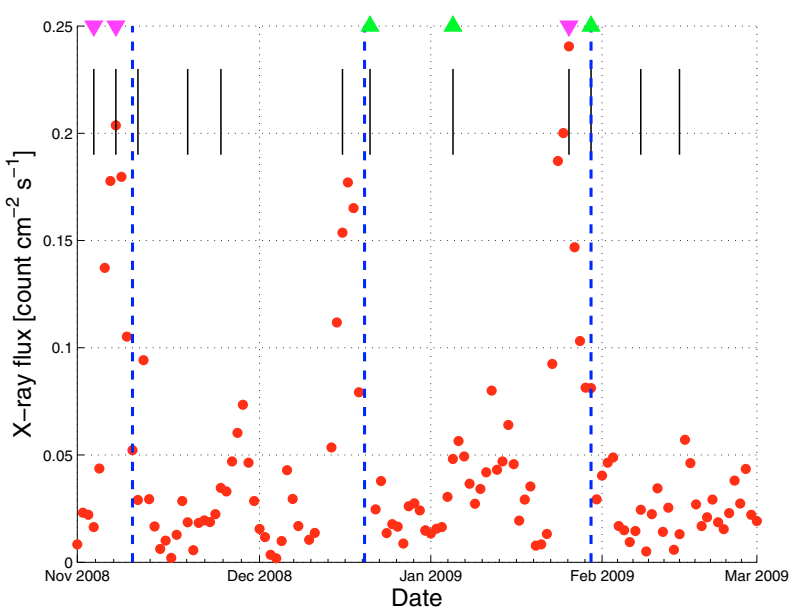

Fig. 1. X-ray lightcurve (Swift/BAT, 15-150 keV) of BP Cru in the period of our observing campaign (red dots). Vertical black lines indicate the epochs of our radio observations, and the dashed blue lines the periastron passages. Green triangles peak up indicate a positive spectral index, magenta triangles peak down indicate negative spectral index.

average $\approx 0.3 \mathrm{mJy}$ (i.e. a detection limit of $\approx 0.9 \mathrm{mJy}$ ) at both frequencies. To prevent any loss of sensitivity on target because of possible artefacts, the ATCA array was pointed \pm 10 arcsec away from BP Cru. Finally, most of the observations were completed in remote mode, by using the exceptionally functional software available for ATCA.

Bandpass, flux, and phase calibration were performed using standard procedures in AIPS $^{1}$ with a ParselTongue ${ }^{2}$ interface to allow quick and equal reduction of many observational epochs, as well as in MIRIAD ${ }^{3}$. The bandpass calibrator was $0823-500$ (1934-638 for epochs 3 and 4). The flux scale was set by the Stokes $I$ fluxes at 4.8 and $8.6 \mathrm{GHz}$ of the phase calibrator 1148 671 , i.e., 1.43 and 0.92 Jy, respectively. All solutions obtained toward the calibrators were applied to the target, and images were

\footnotetext{
1 http://www.aips.nrao.edu/

2 http://www .radionet-eu.org/rnwiki/ParselTongue

3 http://www.atnf.csiro.au/computing/software/miriad/
} 
Table 3. Details of the detections and upper limits for the non-detections of BP Cru ordered by orbital phase.

\begin{tabular}{|c|c|c|c|}
\hline \multirow[t]{2}{*}{ Epoch (phase) ${ }^{a}$} & \multicolumn{2}{|c|}{ Flux $[\mathrm{mJy}]^{b}$} & \multirow[t]{2}{*}{$\alpha^{c g}$} \\
\hline & $4.8 \mathrm{GHz}$ & $8.6 \mathrm{GHz}$ & \\
\hline $1(-7)$ & $1.02(0.17)$ & $0.82(0.18)$ & $-0.38_{-1.10}^{+0.28}$ \\
\hline $9(-4)^{d}$ & $1.01(0.16)$ & $0.51(0.13)$ & $\begin{array}{r}-1.17_{-1.93}^{-0.10} \\
-\end{array}$ \\
\hline $2(-3)$ & $0.75(0.14)$ & $<0.48(0.16)$ & $-0.88^{+0.0}$ \\
\hline $10(+0)$ & $0.70(0.14)$ & $0.72(0.13)$ & $+0.05_{-0.61}^{+0.72}$ \\
\hline $3(+1)^{h}$ & $(0.18)$ & $(0.15)$ & \\
\hline $7(+1)^{e}$ & $0.67(0.13)$ & $0.91(0.14)$ & $+0.53_{-0.07}^{+1.14}$ \\
\hline $8(+16)^{f}$ & $0.51(0.09)$ & $0.63(0.09)$ & $+0.36_{-0.18}^{+0.92}$ \\
\hline
\end{tabular}

a Phase indicates "days from periastron".

${ }^{b}$ Numbers in parenthesis indicate the rms value in the maps.

${ }^{c}$ Spectral index, $\alpha=\log \left(F_{8.6} / F_{4.8}\right) / \log \left(v_{8.6} / v_{4.8}\right)$.

${ }^{d}$ Orbital phase also observed in epoch 6 with a compact configuration, and hence not considered useful for this project.

${ }^{e}$ Orbital phase also observed in epoch 3 without detection. See text.

${ }^{f}$ Note that for this epoch Stokes $Q$ at $8.6 \mathrm{GHz}$ is $0.38 \mathrm{mJy}$, while Stokes $V$ and $U$ were not detected. No polarisation signal was detected at $4.8 \mathrm{GHz}$.

$g$ Maximum and minimum values of the spectral index when adding/subtracting $1 \sigma \mathrm{rms}$ to the listed flux.

${ }^{h}$ As mentioned in the text, we do not detect BP Cru at this epoch.

made using different restoring beam sizes to reflect the different array configurations of ATCA in the different epochs. Our short observations produced elongated beam sizes: the average beam sizes for the epochs with detections (see Table 3 ) were $15 \times 1.5$ and $10 \times 0.8$ arcsec at 4.8 and $8.6 \mathrm{GHz}$, respectively. The integration time on target varied between 36 and 153 min (see Table 2), which allowed us to reach rms levels in the final maps of between 0.16 and $0.09 \mathrm{mJy}^{-1}$ beam $^{-1}$ at $4.8 \mathrm{GHz}$ and between 0.17 and $0.09 \mathrm{mJy}_{\text {beam }}{ }^{-1}$ at $8.6 \mathrm{GHz}$. Even epoch 8 , our longest observation of BP Cru, was too short and covered too short a range of hour angles to allow a safe correction for polarisation leakage. In terms of polarisation, we report here a rough estimate for only one epoch.

\section{Results}

A summary of the detections of BP Cru is presented in Table 3. BP Cru was clearly detected at 4 epochs, corresponding to 4 different orbital phases: two before periastron, one after periastron, and one at periastron. The clearest detection was obtained in our longest integration on-source at epoch 8, 16 days after periastron. The cleaned map at $8.6 \mathrm{GHz}$ is presented in Fig. 2. All other detections are significant to at least $5 \sigma \mathrm{rms}$. We also report two more possible detections at epochs 7 and 9 . The uncertainty in the detections is caused by the same orbital phase being observed twice, with a detection in only one of the two occasions. Epochs 6 and 9 correspond to 4 days before periastron, but only epoch 9 was useful (see Sect. 2); epochs 3 and 7 probed BP Cru one day after periastron but a detection could be seen only in epoch 7. Apart from these uncertainties, the most striking aspect here is that BP Cru seems to emit in the radio regime regardless of the orbital phase and especially when it was least expected, i.e., close to apastron.

Another important observation from Table 3 is that $\alpha$ appears to change from being clearly negative right before periastron to being weakly positive after periastron. As is visible from the minimum and maximum values listed in Table $3, \alpha$ seems to be plausibly positive at and after periastron, while it is possibly

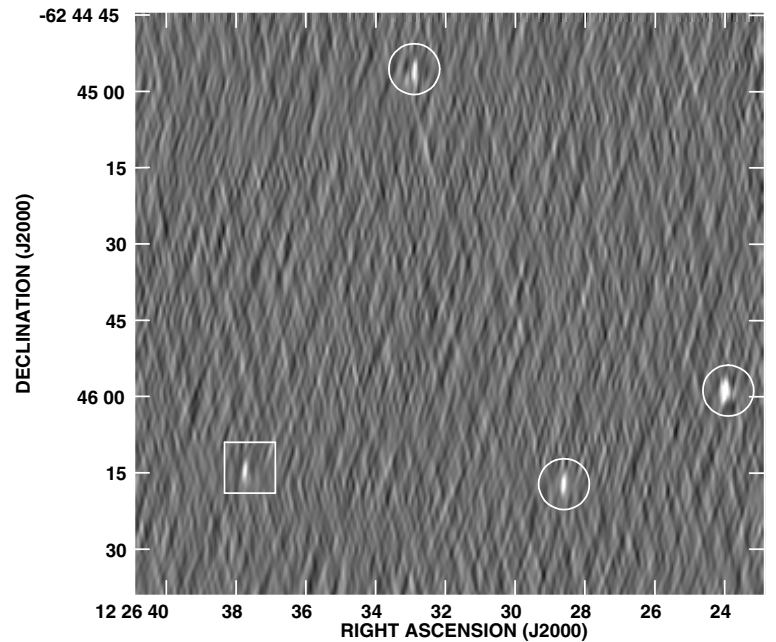

Fig. 2. Stokes $I$ maps of the data taken in epoch 8 , at $8.6 \mathrm{GHz}$. The beam size for this epoch was $3.4 \times 0.7$ arcsec. The box indicates BP Cru, the circles indicate sources A (to the right), B (top in the figure) and $\mathrm{C}$ (lowest in the figure) as in Table 4.

Table 4. Characteristics of the objects detected around BP Cru. Coordinates and fluxes refer to epoch 8 .

\begin{tabular}{ccccc}
\hline \hline & RA(J2000) & Dec(J2000) & $\begin{array}{c}\text { Flux } \\
4.8 / 8.6[\mathrm{mJy}]\end{array}$ & Remarks \\
\hline A & $12: 26: 23.9$ & $-62: 45: 58.75$ & $1.53 / 1.10$ & 2MASS $^{a}$ \\
B & $12: 26: 32.9$ & $-62: 44: 55: 59$ & $1.16 / 0.96$ & $2^{M_{A S S}}$ \\
C & $12: 26: 28.6$ & $-62: 46: 17.58$ & $0.70 / 0.67$ & GSC2.3 $^{c}$ \\
\hline
\end{tabular}

${ }^{a}$ Within $7.5^{\prime \prime} ;{ }^{b}$ within $9.5^{\prime \prime} ;{ }^{c}$ within $1.2^{\prime \prime}$.

negative before that. We note that BP Cru was not detected at $8.6 \mathrm{GHz}$ in epoch 2 , and we therefore consider the $3 \sigma \mathrm{rms}$ level as an upper limit to the flux. Furthermore, it is important to notice that the epochs with possibly negative spectral index correlate with the X-ray outburst, which in turn are known to occur some days before periastron (Watson et al. 1982). Possible explanations of this behaviour are discussed in the next section.

Finally, from polarisation analysis of the longest observation of BP Cru we notice that its radio emission is probably linearly polarised, since Stokes $Q \neq 0$ and Stokes $V=U=0$. This result should be interpreted with caution because our observation at that time was shorter than three hours, barely enough to correct for polarisation leakage between the ATCA orthogonal feeds. Dedicated observations are necessary to retrieve more reliable information about the polarisation of the radio emission from BP Cru.

Several other sources were detected in the ATCA field, which are listed in Table 4. Sources A and B were detected at all useful epochs. Source A appears to lie some 7.5 arcsec away from a 2MASS catalogued object, 12262412-6246060, with $J H K$ magnitudes of $15.83,15.02$, and 18.3 , respectively. Source B lies some 9.5 arcsec from a 2MASS catalogued source, 12263253-6244464, with JHK magnitudes of 15.67, 14.20, and 13.60, respectively. Source C lies at about 1.2 arcsec from an object listed in the Guide Star Catalogue (GSC)2.3 and has a $V$ magnitude of 16.71 . The closest 2 MASS object to $\mathrm{C}$ is 12262821-6246162 at 2.8 arcsec distance, and has JHK magnitudes of $15.03,14.64$, and 14.42, respectively. Taking into account the orientation of the array, and considering the large beam sizes in our observations, our positions of sources $\mathrm{A}-\mathrm{C}$ are 
consistent with those of the catalogued objects mentioned above. The flux densities of these sources remained constant within $\approx 15 \%$. A further discussion of these sources is beyond the scope of this paper.

\section{Discussion}

A spherical stellar wind from Wray 977 is expected to generate continuum fluxes of 1.9 and $2.6 \mathrm{mJy}$ at 4.8 and $8.6 \mathrm{GHz}$, respectively, assuming a distance of $3 \mathrm{kpc}$ for Wray 977 (Panagia \& Felli 1975; Wright \& Barlow 1975; Scuderi et al. 1998), which is more than a factor of two higher flux than we detect. There are several ways in which the predicted fluxes can be reconciled with the observed fluxes. Firstly, there are large uncertainties in the distance to BP Cru, and a distance of $4-5 \mathrm{kpc}$, that is sufficient to decrease our observed fluxes by a factor of two, is perfectly compatible with the most recent estimates by Kaper et al. (2006). Secondly, it is not clear that Wray 977 emits a sufficient number of Lyman photons to ensure that the wind remains completely ionised. In fact, using the results of Scuderi et al. (1998), we estimate that Wray 977 produces some 3-5 times fewer photons than required to keep the entire wind ionised. We also note that the radio flux from a stellar wind can naturally vary by a factor of two (Scuderi et al. 1998).

Typically, the radio emission from the stellar wind of an early-type star has a positive spectral index (Scuderi et al. 1998). We therefore suggest that the radio emission at epochs 7 and 8 is caused by the stellar wind from Wray 977. Consequently, the increased radio flux at $4.8 \mathrm{GHz}$ at the time of the X-ray outburst is probably due to a transient, non-thermal component with a negative spectral index. There is insufficient data to confirm whether this component is most strongly correlated with the orbital phase or the timing of the X-ray outburst.

We propose that the transient non-thermal component appearing during the X-ray outburst, originates from a weak and short-lived (relativistic?) jet, as in a microquasar. To test this hypothesis, we isolated the transient component by subtracting the flux from epoch 8 that from epoch 1 . The transient component then has fluxes of 0.51 and $0.19 \mathrm{mJy}$ at 4.8 and $8.6 \mathrm{GHz}$, respectively, and a spectral index $\alpha=-1.7_{-7.0}^{+0.1}$. This suggests a somewhat steeper index than is usually seen in microquasars (e.g., McClintock et al. 2009; Trushkin et al. 2008), in which $\alpha>$ -1 , but the uncertainties are so large that no firm conclusions can be drawn. We also compare BP Cru with the microquasar GRS 1915+105. During an extended period in 2008, Swift/BAT registered an X-ray flux of $0.1 \mathrm{ph} \mathrm{cm}^{-2} \mathrm{~s}^{-1}$ from GRS 1915+105, and at the same time its radio flux at $4.8 \mathrm{GHz}$ remained above $100 \mathrm{mJy}$ (Trushkin et al. 2008). The X-ray flux from BP Cru at the peak of an outburst is even higher, $0.2 \mathrm{ph} \mathrm{cm}^{-2} \mathrm{~s}^{-1}$, but the flux of its transient radio component is at least 200 times fainter than that of GRS $1915+105$.

A problem with this interpretation is that the detection at epoch 1 occurs so close to the onset of the X-ray outburst that one might question whether a jet may have already formed at that time. An alternative source of the non-thermal electrons would be a shock that forms in the stellar wind as the neutron star approaches Wray 977 and distorts the stellar wind throughout its gravitational field.
In the future, one should follow the evolution of the radio flux from BP Cru over all orbital phases, and every observation should be sufficiently long to allow the study of the polarisation of the radio emission. This is readily possible using the Compact Array Broadband Backend (CABB), newly installed at ATCA. Its wide band increases the overall sensitivity by a factor 4 , ensuring the detection of BP Cru at any orbital phase. Also, with sufficiently long integrations to provide good signal-to-noise ratios, it will be possible to obtain a full spectrum in two bands with ideally one point every $250 \mathrm{MHz}$.

\section{Summary and conclusions}

We summarise our findings as follows:

- We have presented the first detection of the high-mass X-ray binary BP Cru in centimetre radio continuum at both 4.8 and $8.6 \mathrm{GHz}$. The source was detected several times at different orbital phases, indicating that BP Cru emits radio waves independently of its orbital phase.

- The radio emission from BP Cru shows a varying spectral index, suggesting that it is the superposition of two components: a persistent thermal emission from the wind of the B hypergiant mass donor Wray 977, and an episodic emission, perhaps a weak jet, that appears at the time of the X-ray outburst.

- Further observations using the newly installed Compact Array Broadband Backend (CABB) will greatly improve both the estimate of the spectral index and the information about the polarisation of the radio emission of BP Cru.

Acknowledgements. We thank the referee, Roland Walter, for his comments that clearly improved the quality of our paper. M.P. and U.T. thank the Swedish National Research Council for travel support to allow observations in situ at ATCA. M.P. thanks the staff at ATCA for their support during the stay in Narrabri, during the sessions of remote observing and for a generous time allocation. M.P. is supported by a two-year postdoc from the University of Gothenburg (Sweden). Swift/BAT transient monitor results provided by the Swift/BAT team. This research has made use of the SIMBAD data base operated at CDS, Strasbourg France, and NASA's Astrophysics Data System.

\section{References}

Duldig, M. L., Greenhill, J. G., Thomas, R. M., et al. 1979, MNRAS, 187, 567 Harmon, B. A., Wilson, C. A., Zhang, S. N., et al. 1995, Nature, 374, 703

Kaper, L., van der Meer, A., \& Najarro, F. 2006, A\&A, 457, 595

Koh, D. T., Bildsten, L., Chakrabarty, D., et al. 1997, ApJ, 479, 933

Kreykenbohm, I., Wilms, J., Coburn, W., et al. 2004, A\&A, 427, 975

Leahy, D. A., \& Kostka, M. 2008, MNRAS, 384, 747

McClintock, J. E., Remillard, R. A., Rupen, M. P., et al. 2009, ApJ, 698, 1398

Nelson, R. F., \& Spencer, R. E. 1988, MNRAS, 234, 1105

Panagia, N., \& Felli, M. 1975, A\&A, 39, 1

Pravdo, S. H., Day, C. S. R., Angelini, L., et al. 1995, ApJ, 454, 872

Scuderi, S., Panagia, N., Stanghellini, C., Trigilio, C., \& Umana, G. 1998, A\&A, 332,251

Trushkin, S., Nizhelskij, N. A., \& Bursov, N. N. 2008, ATel

Watson, M. G., Warwick, R. S., \& Corbet, R. H. D. 1982, MNRAS, 199, 915

White, N. E., Mason, K. O., Huckle, H. E., Charles, P. A., \& Sanford, P. W. 1976, ApJ, 209, L119

Wright, A. E., \& Barlow, M. J. 1975, MNRAS, 170, 41 